\title{
FNDC3B is associated with ER stress and poor prognosis in cervical cancer
}

\author{
BING HAN $^{1}$, HONGBO WANG ${ }^{1}$, JIANZHAO ZHANG ${ }^{2}$ and JINGWEI TIAN ${ }^{1}$ \\ ${ }^{1}$ Key Laboratory of Molecular Pharmacology and Drug Evaluation, School of Pharmacy, Ministry of Education, \\ Collaborative Innovation Center of Advanced Drug Delivery System and Biotech Drugs in Universities of Shandong; \\ ${ }^{2}$ College of Life Sciences, Yantai University, Yantai, Shandong 264005, P.R. China
}

Received March 17, 2019; Accepted September 20, 2019

DOI: $10.3892 / \mathrm{ol} .2019 .11098$

\begin{abstract}
Currently, the occurrence and mortality rate of cervical cancer is high, particularly in low-to-middle-income countries. Therefore, the development of novel diagnostic and treatment strategies for cervical cancer is urgently required. The aim of the present study was to assess the prognostic significance of fibronectin type III domain containing $3 \mathrm{~B}$ (FNDC3B) expression in patients with cervical cancer and to determine the underlying mechanism of FNDC3 in tumor development. Analysis of the ONCOMINE database revealed that FNDC3B was significantly upregulated in cervical cancer tissue compared with normal tissue. Additionally, FNDC3B expression data and the clinical characteristics of patients with cervical cancer were obtained from the cBioPortal database. Correlations between FNDC3B expression and overall survival were subsequently investigated. The results revealed that increased FNDC3B expression was significantly correlated with a lower overall survival in patients with cervical cancer. A co-expression network was subsequently constructed to elucidate the function of FNDC3B in cervical cancer. Co-expression genes for FNDC3B were obtained from the cBioPortal database and were subjected to Gene Ontology and Kyoto Encyclopedia of Genes and Genomes analyses. The results demonstrated that the genes were enriched in pathways associated with migration,
\end{abstract}

Correspondence to: Professor Jianzhao Zhang, College of Life Sciences, Yantai University, 32 Qingquan Avenue Yantai, Shandong 264005, P.R. China

E-mail: zhangjianzhao@163.com

Professor Jingwei Tian, Key Laboratory of Molecular Pharmacology and Drug Evaluation, School of Pharmacy, Ministry of Education, Collaborative Innovation Center of Advanced Drug Delivery System and Biotech Drugs in Universities of Shandong, Yantai University, 32 Qingquan Avenue, Yantai, Shandong 264005, P.R. China E-mail: tianjingwei@luye.cn

Key words: fibronectin type III domain containing 3B, prognostic biomarker, endoplasmic reticulum stress, unfolded protein response, Gene Ontology, Kyoto Encyclopedia of Genes and Genomes invasion, endoplasmic reticulum (ER) stress and the unfolded protein response (UPR). Furthermore, immunofluorescence results obtained from the Human Protein Atlas revealed that the FNDC3B protein was localized to the ER. The results revealed that upregulated FNDC3B expression may be a biomarker for poor prognosis for patients with cervical cancer. Additionally, the results revealed that FNDC3B may serve an oncogenic role in cancer development via ER stress, UPR, cell migration and invasion. However, further studies are required to determine the exact molecular mechanism of FNDC3B in the development of cervical cancer and to assess its potential as a novel therapeutic target for the treatment of this disease.

\section{Introduction}

Cervical cancer is the fourth most frequently diagnosed tumor and the fourth leading cause of cancer-associated mortalities in women worldwide. In 2018, 570,000 females were diagnosed with cervical cancer and $\sim 311,000$ deaths were reported (1). The burden of this type of cancer remains heavy, particularly in low-to-middle-income countries. In fact, the number of cervical cancer deaths in developing countries accounted for $\sim 90 \%$ of all cervical cancer deaths worldwide in 2015 (2). Cervical cancer ranks second in incidence and mortality in low- and middle-income countries (1). In high-income countries, the incidence and mortality rates of cervical cancer have decreased dramatically due to screening programs being made available in the mid-20th century (3). From 2006 to 2014 in the United States of America (USA), delay-adjusted cervical cancer incidence rates decreased at an average annual percentage rate of $0.3 \%$ (4). Mortality rates have also declined at an average annual rate of $0.8 \%$ between 2003 and 2014 (4). However, in 2018, 13,240 women were diagnosed with invasive cervical cancer and 4,170 patients succumbed to the disease in the USA (5). Human papilloma virus infection is a risk factor for cervical cancer, but infection alone does not necessarily lead to the development of the disease (6). Thus, the identification of novel biomarkers with prognostic value is urgently required. Additionally, this may clarify the mechanism underlying tumorigenesis and aid the identification of novel therapeutic targets. 
Fibronectin type III domain containing 3B (FNDC3B), also termed factor for adipocyte differentiation 104 (FAD104), was initially determined to be a regulator of adipocyte differentiation (7). A previous study used gene targeting to demonstrate that FNDC3B was involved in cell proliferation, adhesion, spreading and migration in FNDC3B-deficient mice (8). FNDC3B has been previously identified as an oncogene that promotes cell migration in hepatocellular carcinoma $(3,9)$. However, to the best of the authors' knowledge, the prognostic value and function of FNDC3B in cervical cancer has not yet been elucidated.

Systematic biology comprehensively determines the underlying mechanism and allows the identification of new biomarkers in human disease on a global scale. Networks are practical graphical representations of complex interactions (10). The combination of systematic biology and networks is therefore useful to visualize complex biological activities and to annotate protein functions and predictions (11). Thus, the present study utilized systematic biology and network methods to predict the effect of FNDC3B expression on the prognosis of patients with cervical carcinoma and to annotate protein function.

The present study assessed the expression of FNDC3B mRNA in patients with cervical cancer using the ONCOMINE database. Subsequently, the association between FNDC3B expression and prognosis was investigated, and the biological function and mechanism of action of FNDC3B in patients with cervical cancer was explored using publicly accessible databases.

\section{Materials and methods}

Expression analysis of $F N D C 3 B$ in cervical cancer. The expression value of FNDC3B mRNA in cervical cancer was analyzed using the ONCOMINE database (version 4.5; www. oncomine.org/resource/login.html) (12). Cancerous tissues and normal tissues obtained from healthy volunteers were subsequently compared according to the default settings of $\mathrm{P}<1 \times 10^{-4}$, fold-change $>2$ and gene ranking in the top $10 \%$ (13)

Survival analysis. FNDC3B gene expression data and the clinical characteristics of patients with cervical cancer were downloaded from The Cancer Genome Atlas (www.cbioportal. org) $(14,15)$. The association between FNDC3B expression and patient overall survival (OS) was analyzed using the $\mathrm{R}$ package survival (version 2.43-3, https://cran.r-project. org/web/views/Survival.html) $(16,17)$. Samples were then divided into high- and low-expression groups using the median expression level of FNDC3B mRNA as the cut-off point. The difference in OS between the two groups was assessed using Kaplan-Meier curves followed by a log-rank test.

Co-expression gene identification and protein-protein interaction network visualization. The cBioportal database (cbioportal.org) was used to assess and visualize cancer co-expression data (14), which was subsequently downloaded. FNDC3B co-expression genes with an absolute correlation coefficient of $>0.4$ and $\mathrm{P}<0.05$ were obtained from cBioPortal. The Search Tool for the Retrieval of Interacting Genes/Proteins (STRING version 10.5, string-db. org) was used to perform protein-protein interaction (PPI) analysis (18). Data was subsequently downloaded and the PPI network was constructed using Cytoscape software (version 3.7.1) (19).

Gene ontology (GO) and Kyoto encyclopedia of genes and genomes (KEGG) enrichment analysis. The clusterProfiler package (version 3.8.1, http://bioconductor. org/packages/release/bioc/html/clusterProfiler.html) (20) in R was used to identify and visualize the GO terms (geneontology. org) and KEGG pathways (www.genome.jp/kegg) associated with the FNDC3B co-expression genes. The P-value was adjusted using the Benjamini-Hochberg method. $\mathrm{P}<0.05$ and $\mathrm{q}<0.05$ were set as the cut-off criteria for significant enrichment.

Localization of FNDC $3 B$ in cells. The cellular localization of FNDC3B was determined using The Human Protein Atlas (version 18.1, www.proteinatlas.org) (21). The key word used for searching was 'FNDC3B'. The location of FNDC3B in cells was determined using the immunofluorescence with anti-FNDC3B antibodies (cat. no. HPA007859; Atlas Antibodies AB). Images were obtained from www.proteinatlas.org/ENSG00000075420-FNDC3B/cell\#img.

Statistical analysis. Statistical analyses were performed using R software (version 3.5.1; R Foundation for Statistical Computing). The relative expression of FNDC3B was presented as the mean \pm standard deviation. The differential expression of FNDC3B between cancerous and non-cancerous samples was compared using an independent Student's t-test. A total of 20 cancerous and eight non-cancerous samples from the multi-cancer dataset published by Pyeon et al (22) were selected for analysis in the present study. An additional 32 cancerous and 21 non-cancerous samples were selected from a dataset published by Scotto et al (23). Kaplan-Meier survival analysis was performed to estimate the survival distributions and the log-rank test was used to compare the survival curves. The correlation of gene expression was analyzed by Spearman's correlation test. $\mathrm{P}<0.05$ was considered to indicate a statistically significant difference.

\section{Results}

FNDC3B expression is upregulated in cervical cancer. Analysis of the ONCOMINE database revealed that the level of FNDC3B mRNA was significantly increased in cervical cancer tissues compared with normal tissues. By contrast, no cervical cancer tissues with downregulated FNDC3B expression were identified (Fig. 1).

Survival prediction of FNDC3B in cervical cancer. Survival analysis was performed to investigate the association between upregulated FNDC3B expression and the clinical outcome of patients with cervical cancer. As presented in Fig. 2, upregulated FNDC3B expression was significantly associated with a lower OS in patients with cervical cancer. The results indicated that upregulated FNDC3B expression may serve as a biomarker of poor prognosis in patients with cervical cancer. 
A Comparison of FNDC3B across 2 analyses Upregulation

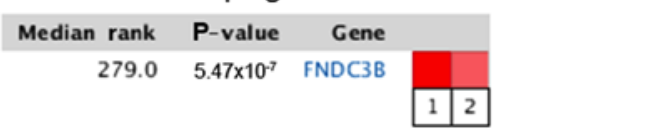

Legend

1. Cervical Cancer vs. Normal

Pyeon Multi-cancer, Cancer Res, 2007

2. Cervical Squamous Cell Carcinoma vs. Normal

Scotto Cervix 2, Genes

Chromosomes Cancer, 2008

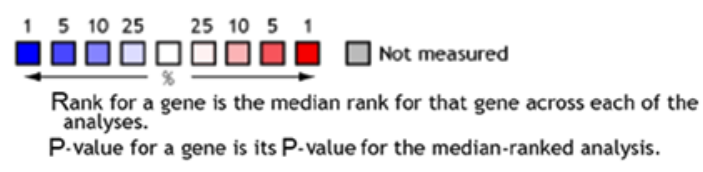

C Scotto Cervix 2 statistics

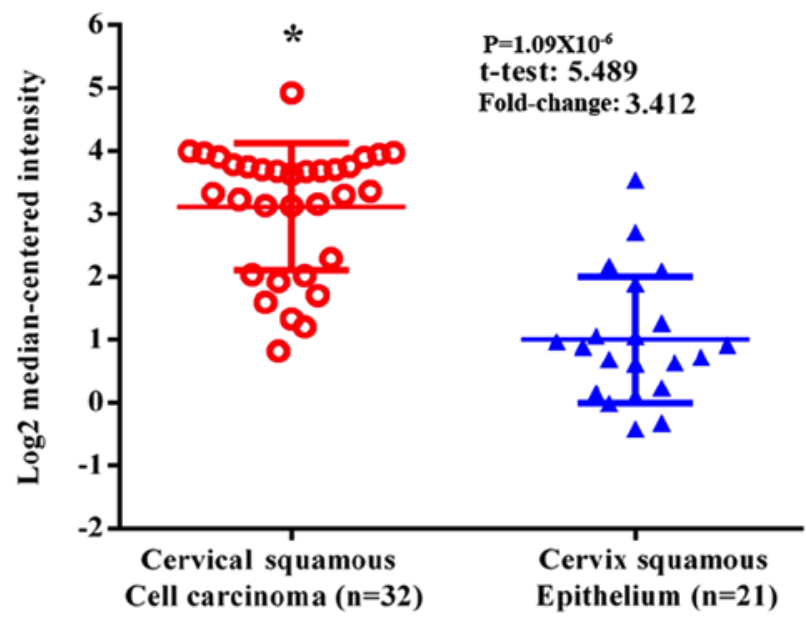

B Pyeon Multi-cancer statistics

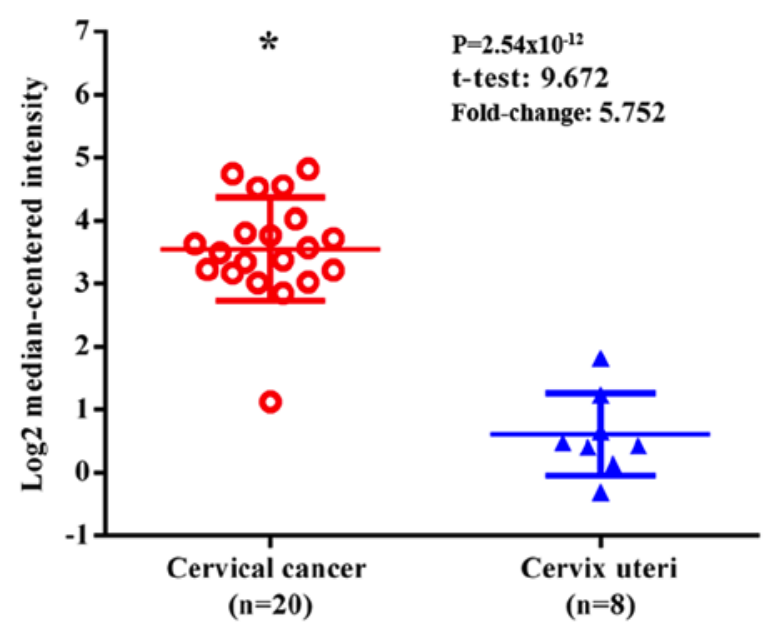

Figure 1. Validation of upregulated FNDC3B expression using the ONCOMINE database. FNDC3B mRNA expression in cancerous and corresponding normal tissue was determined using the ONCOMINE database. (A) The comparison of FNDC3B expression across two cervical cancer analyses is presented. Red and blue represent upregulated and downregulated expression, respectively. (B) FNDC3B expression in cervical cancer and normal tissue samples from the multi-cancer study by Pyeon et al (22) (normal tissues, 8 cases; cervical cancer tissues, 20 cases). (C) FNDC3B expression in cervical cancer and normal tissue samples from the cervical cancer study by Scotto et al (23) (normal tissues, 21 cases; cervical cancer tissues, 32 cases). Data are presented as the mean $\pm \mathrm{SD}$. ${ }^{*} \mathrm{P}<0.05$ vs. the non-cancerous group. FNDC3B, fibronectin type III domain containing 3B.

Co-expression gene identification and PPI network visualization. Analysis of the cBioPortal database revealed that a total of 88 genes were significantly co-expressed with FNDC3B. Additionally, 79 co-expressed genes were positively correlated with FNDC3B and 9 co-expressed genes were negatively correlated with FNDC3B (Table I). A PPI network consisting of FNDC3B co-expression genes based on the STRING database was constructed using Cytoscape software. The co-expression network contained 66 nodes and 179 edges (Fig. 3).

Gene co-expression network analysis is associated with FNDC3B in cervical cancer. The results of GO enrichment analysis revealed that the co-expression genes were significantly enriched in 72 biological processes (BPs), 29 molecule functions (MFs) and 50 cellular components (CCs). The five top ranked BPs, MFs and CCs were as follows: 'Extracellular matrix organization', 'extracellular structure organization', 'protein folding', 'response to unfolded protein', 'response to topologically

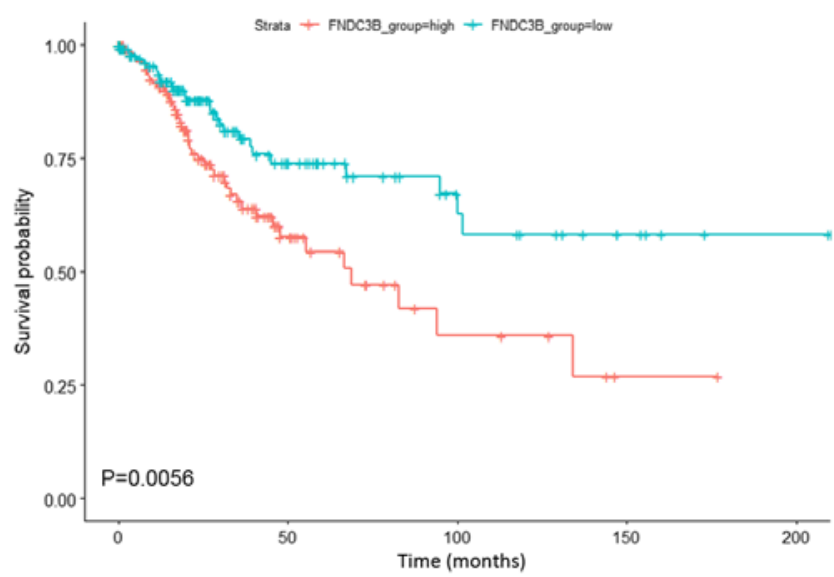

Figure 2. Overall survival analysis of FNDC3B with data obtained from The Cancer Genome Atlas database. Overall survival analysis of FNDC3B was assessed using Kaplan-Meier curves followed by a log-rank test. The blue and red lines represent patients with a low and FNDC3B expression, respectively. FNDC3B, fibronectin type III domain containing 3B. 
Table I. Co-expressed genes associated with fibronectin type III domain containing $3 \mathrm{~B}$.

\begin{tabular}{|c|c|c|c|}
\hline Correlated gene & Cytoband & Spearman's correlation coefficient & P-value \\
\hline NCEH1 & $3 q 26.31$ & 0.56 & $3.51 \times 10^{-26}$ \\
\hline B4GALT1 & $9 \mathrm{p} 21.1$ & 0.54 & $5.53 \times 10^{-25}$ \\
\hline CALU & $7 q 32.1$ & 0.54 & $7.49 \times 10^{-25}$ \\
\hline LAMC1 & $1 \mathrm{q} 25.3$ & 0.54 & $1.87 \times 10^{-24}$ \\
\hline ITGB1 & $10 p 11.22$ & 0.52 & $4.80 \times 10^{-23}$ \\
\hline MCFD2 & $2 \mathrm{p} 21$ & 0.52 & $2.19 \times 10^{-22}$ \\
\hline TMED7 & $5 q 22.3$ & 0.51 & $6.07 \times 10^{-22}$ \\
\hline COPB2 & $3 q 23$ & 0.51 & $8.75 \times 10^{-22}$ \\
\hline SKIL & $3 q 26.2$ & 0.51 & $1.72 \times 10^{-21}$ \\
\hline UGGT1 & $2 q 14.3$ & 0.50 & $3.66 \times 10^{-21}$ \\
\hline TMEM263 & $12 q 23.3$ & 0.50 & $5.60 \times 10^{-21}$ \\
\hline HSPA5 & $9 q 33.3$ & 0.50 & $2.20 \times 10^{-20}$ \\
\hline SEC62 & $3 q 26.2$ & 0.49 & $4.63 \times 10^{-20}$ \\
\hline SUSD1 & $9 q 31.3-q 32$ & 0.49 & $1.62 \times 10^{-19}$ \\
\hline PLOD2 & $3 q 24$ & 0.48 & $2.13 \times 10^{-19}$ \\
\hline TEAD1 & $11 \mathrm{p} 15.3$ & 0.47 & $2.76 \times 10^{-18}$ \\
\hline LMAN1 & $18 \mathrm{q} 21.32$ & 0.47 & $2.81 \times 10^{-18}$ \\
\hline HSP90B1 & $12 \mathrm{q} 23.3$ & 0.47 & $3.08 \times 10^{-18}$ \\
\hline FKBP14 & $7 \mathrm{p} 14.3$ & 0.47 & $6.05 \times 10^{-18}$ \\
\hline ITGB3 & $17 q 21.32$ & 0.47 & $6.39 \times 10^{-18}$ \\
\hline CCDC50 & $3 q 28$ & 0.47 & $6.82 \times 10^{-18}$ \\
\hline KIRREL1 & $1 q 23.1$ & 0.47 & $6.89 \times 10^{-18}$ \\
\hline LPP & $3 q 27.3-q 28$ & 0.47 & $7.66 \times 10^{-18}$ \\
\hline SLC39A14 & $8 \mathrm{p} 21.3$ & 0.46 & $1.04 \times 10^{-17}$ \\
\hline NCKAP5L & $12 q 13.12$ & 0.46 & $1.12 \times 10^{-17}$ \\
\hline ATP13A3 & $3 q 29$ & 0.46 & $1.29 \times 10^{-17}$ \\
\hline EXT2 & $11 \mathrm{p} 11.2$ & 0.46 & $1.76 \times 10^{-17}$ \\
\hline LAMB1 & 7q31.1 & 0.46 & $2.85 \times 10^{-17}$ \\
\hline SLC33A1 & $3 q 25.31$ & 0.46 & $2.99 \times 10^{-17}$ \\
\hline TTYH3 & $7 \mathrm{p} 22.3$ & 0.46 & $3.22 \times 10^{-17}$ \\
\hline FSTL1 & $3 q 13.33$ & 0.46 & $4.40 \times 10^{-17}$ \\
\hline SSR3 & $3 q 25.31$ & 0.46 & $4.76 \times 10^{-17}$ \\
\hline IKBIP & $12 \mathrm{q} 23.1$ & 0.45 & $6.75 \times 10^{-17}$ \\
\hline SERPINH1 & $11 \mathrm{q} 13.5$ & 0.45 & $2.10 \times 10^{-16}$ \\
\hline PDIA6 & $2 \mathrm{p} 25.1$ & 0.44 & $4.18 \times 10^{-16}$ \\
\hline TMEM30A & $6 q 14.1$ & 0.44 & $4.84 \times 10^{-16}$ \\
\hline PLOD1 & $1 \mathrm{p} 36.22$ & 0.43 & $1.57 \times 10^{-15}$ \\
\hline PLBD2 & $12 q 24.13$ & 0.43 & $1.63 \times 10^{-15}$ \\
\hline AGRN & $1 \mathrm{p} 36.33$ & 0.43 & $1.75 \times 10^{-15}$ \\
\hline GNS & $12 q 14.3$ & 0.43 & $1.96 \times 10^{-15}$ \\
\hline ZNF281 & $1 q 32.1$ & 0.43 & $2.00 \times 10^{-15}$ \\
\hline SLC41A2 & $12 q 23.3$ & 0.43 & $3.07 \times 10^{-15}$ \\
\hline ADAM9 & $8 p 11.22$ & 0.43 & $3.18 \times 10^{-15}$ \\
\hline TGFBR2 & $3 \mathrm{p} 24.1$ & 0.43 & $3.54 \times 10^{-15}$ \\
\hline HIF1A & $14 q 23.2$ & 0.43 & $3.80 \times 10^{-15}$ \\
\hline STC1 & $8 \mathrm{p} 21.2$ & 0.43 & $4.46 \times 10^{-15}$ \\
\hline DNAJC10 & $2 q 32.1$ & 0.43 & $5.28 \times 10^{-15}$ \\
\hline ITGAV & $2 q 32.1$ & 0.43 & $7.41 \times 10^{-15}$ \\
\hline GANAB & $11 \mathrm{q} 12.3$ & 0.42 & $9.71 \times 10^{-15}$ \\
\hline PAPSS2 & $10 \mathrm{q} 23.2-\mathrm{q} 23.31$ & 0.42 & $1.02 \times 10^{-14}$ \\
\hline RAB43 & $3 q 21.3$ & 0.42 & $1.05 \times 10^{-14}$ \\
\hline TGOLN2 & $2 \mathrm{p} 11.2$ & 0.42 & $1.63 \times 10^{-14}$ \\
\hline
\end{tabular}


Table I. Continued.

\begin{tabular}{|c|c|c|c|}
\hline Correlated gene & Cytoband & Spearman's correlation coefficient & P-value \\
\hline TMEM39A & $3 q 13.33$ & 0.42 & $1.71 \times 10^{-14}$ \\
\hline ITFG1 & $16 \mathrm{q} 12.1$ & 0.42 & $2.29 \times 10^{-14}$ \\
\hline BICC1 & $10 \mathrm{q} 21.1$ & 0.42 & $2.66 \times 10^{-14}$ \\
\hline ZBTB38 & $3 q 23$ & 0.41 & $3.83 \times 10^{-14}$ \\
\hline ERLEC1 & $2 \mathrm{p} 16.2$ & 0.41 & $5.09 \times 10^{-14}$ \\
\hline SEC24D & $4 q 26$ & 0.41 & $5.18 \times 10^{-14}$ \\
\hline HSPA13 & $21 \mathrm{q} 11.2$ & 0.41 & $5.49 \times 10^{-14}$ \\
\hline LATS2 & $13 q 12.11$ & 0.41 & $5.53 \times 10^{-14}$ \\
\hline MPDZ & $9 \mathrm{p} 23$ & 0.41 & $5.81 \times 10^{-14}$ \\
\hline LAMC2 & $1 q 25.3$ & 0.41 & $5.85 \times 10^{-14}$ \\
\hline OSMR & $5 \mathrm{p} 13.1$ & 0.41 & $6.70 \times 10^{-14}$ \\
\hline RAI14 & $5 \mathrm{p} 13.2$ & 0.41 & $7.52 \times 10^{-14}$ \\
\hline HSPG2 & $1 \mathrm{p} 36.12$ & 0.41 & $7.53 \times 10^{-14}$ \\
\hline PDIA4 & $7 \mathrm{q} 36.1$ & 0.41 & $7.92 \times 10^{-14}$ \\
\hline ITGA1 & $5 q 11.2$ & 0.41 & $9.88 \times 10^{-14}$ \\
\hline CMTM6 & $3 p 22.3$ & 0.41 & $1.02 \times 10^{-13}$ \\
\hline SURF4 & $9 \mathrm{q} 34.2$ & 0.41 & $1.25 \times 10^{-13}$ \\
\hline TNS3 & $7 \mathrm{p} 12.3$ & 0.41 & $1.29 \times 10^{-13}$ \\
\hline CPD & $17 \mathrm{q} 11.2$ & 0.41 & $1.40 \times 10^{-13}$ \\
\hline OSBPL10 & $3 \mathrm{p} 23$ & 0.41 & $1.42 \times 10^{-13}$ \\
\hline CD276 & $15 \mathrm{q} 24.1$ & 0.40 & $2.47 \times 10^{-13}$ \\
\hline GALNT1 & $18 \mathrm{q} 12.2$ & 0.40 & $2.72 \times 10^{-13}$ \\
\hline CKAP4 & $12 \mathrm{q} 23.3$ & 0.40 & $2.91 \times 10^{-13}$ \\
\hline GPX8 & $5 q 11.2$ & 0.40 & $2.97 \times 10^{-13}$ \\
\hline NEDD9 & $6 \mathrm{p} 24.2$ & 0.40 & $3.25 \times 10^{-13}$ \\
\hline TGFB2 & $1 \mathrm{q} 41$ & 0.40 & $3.30 \times 10^{-13}$ \\
\hline PARVA & $11 \mathrm{p} 15.3$ & 0.40 & $3.37 \times 10^{-13}$ \\
\hline NUDT8 & $11 \mathrm{q} 13.2$ & -0.40 & $3.28 \times 10^{-13}$ \\
\hline NOL12 & 22q13.1 & -0.40 & $3.28 \times 10^{-13}$ \\
\hline TIMM13 & 19p13.3 & -0.40 & $3.08 \times 10^{-13}$ \\
\hline ENDOG & $9 \mathrm{q} 34.11$ & -0.40 & $2.91 \times 10^{-13}$ \\
\hline COQ4 & $9 q 34.11$ & -0.41 & $1.41 \times 10^{-13}$ \\
\hline VPS28 & $8 \mathrm{q} 24.3$ & -0.41 & $6.90 \times 10^{-14}$ \\
\hline CYC1 & $8 \mathrm{q} 24.3$ & -0.43 & $4.54 \times 10^{-15}$ \\
\hline COMTD1 & $10 \mathrm{q} 22.2$ & -0.43 & $3.48 \times 10^{-15}$ \\
\hline NDUFS7 & 19p13.3 & -0.43 & $1.82 \times 10^{-15}$ \\
\hline
\end{tabular}

incorrect protein', 'unfolded protein binding', 'extracellular matrix binding, 'protein disulfide isomerase activity', 'intramolecular oxidoreductase activity, transposing S-S bonds', 'chemokine binding', 'endoplasmic reticulum (ER) lumen', 'melanosome', 'pigment granule', 'ER chaperone complex' and 'ER-Golgi intermediate compartment' (Fig. 4A-C). The results of KEGG pathway enrichment analysis demonstrated that the co-expression genes of FNDC3B were significantly enriched in four pathways, including 'protein processing in ER', 'extracellular matrix (ECM)-receptor interaction', 'focal adhesion' and 'PI3K-Akt signaling pathway' (Fig. 4D).

Cellular location of FNDC3B. The results of immunofluorescence analysis obtained from The Human Protein
Atlas database are presented in Fig. 5. Co-localization of FNDC3B (green) and ER (yellow) was observed, indicating that FNDC3B was localized to the ER.

\section{Discussion}

The present study assessed the prognostic effect of FNDC3B and its potential underlying molecular mechanisms in cervical cancer using bioinformatics tools. FNDC3B is an important oncogenic driver gene that was identified in an oncogenomic screen for oncogenes in hepatocellular carcinoma (3). Lin et al (9) identified FNDC3B as a biomarker and therapeutic target for hepatocellular carcinoma metastasis. In the present study, FNDC3B expression was upregulated in cervical cancer 


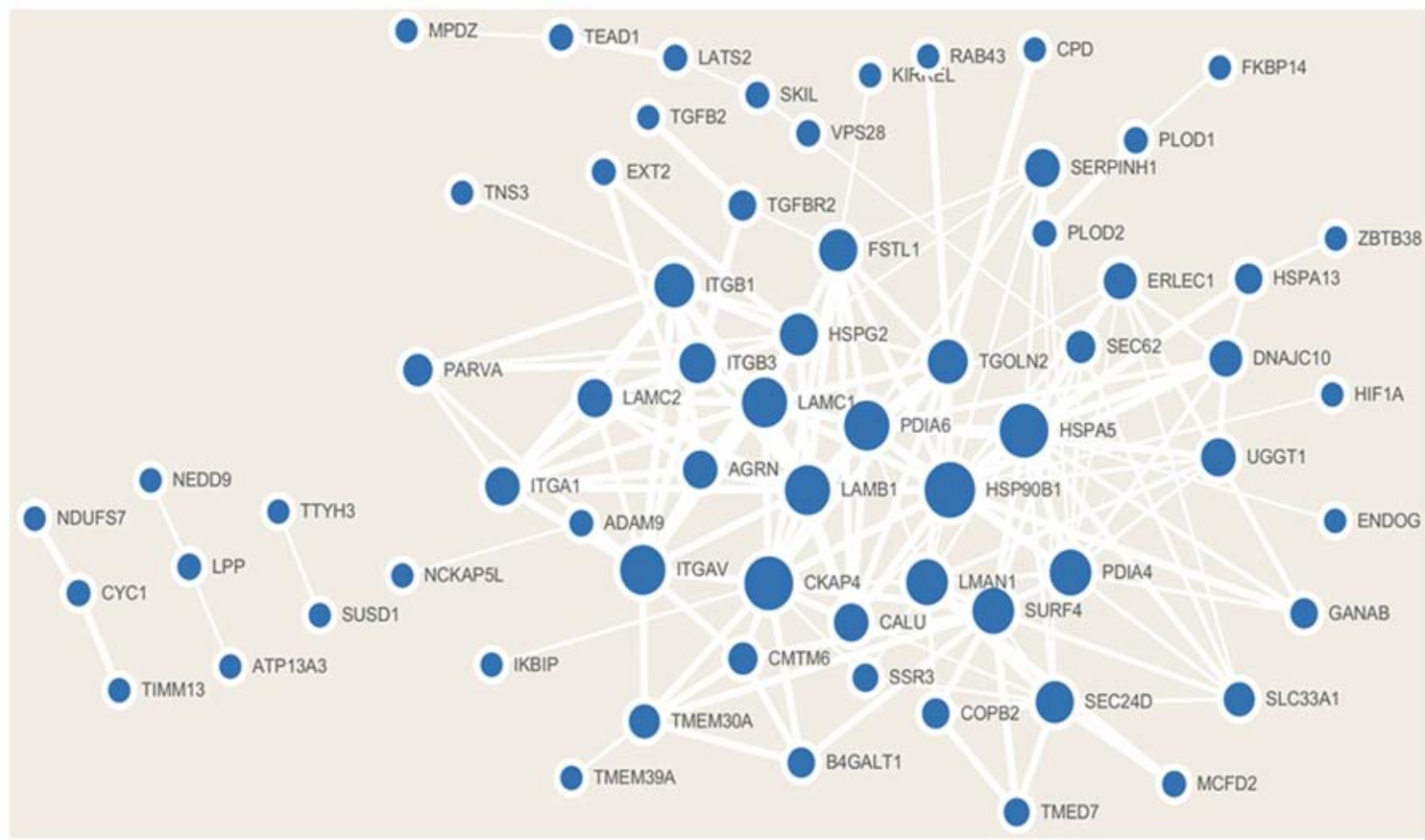

Figure 3. Visualization of the PPI network of FNDC3B co-expression. In the PPI, co-expressed genes are presented as nodes and the interactions between them are presented as edges. Label size indicates the degree value and the thickness of the lines represents the level of closeness between the two nodes. PPI, protein-protein interaction; FNDC3B, fibronectin type III domain containing 3B.

tissues and was associated with a poor prognosis. As the function of FNDC3B in cervical cancer is unknown, the present study investigated its potential functions by constructing a co-expression network. GO and KEGG enrichment analyses revealed that FNDC3B was associated with ER stress and UPR signaling. Furthermore, KEGG pathways analysis revealed that FNDC3B was enriched in 'protein processing in ER'. The ER is a subcellular organelle that is associated with protein synthesis, folding and quality control (24). Adequately folded proteins are subsequently transported to their destined sites, whereas terminally misfolded proteins are subjected to degradation via ER-associated degradation pathways (25). Certain biological processes including 'protein folding', 'response to unfolded protein' and 'response to topologically incorrect protein' were enriched in the present study and were associated with ER stress and UPR activation. The highest enrichment of MF and CC were 'unfolded protein binding' and 'ER lumen', respectively. These indicated that the function of FNDC3B may be associated with ER stress and UPR. The role of ER stress and UPR activation in the development of cancer has been previously revealed in various types of cancer, including cervical cancer (26-28). Tumor growth can produce several cell-intrinsic and extrinsic stresses (29). The effects induced by these stresses disrupt the ER protein-folding environment, resulting in protein misfolding and the accumulation of misfolded proteins, which is referred to as ER stress (26). Tolerable levels of ER stress promote tumor development by bolstering viability under hypoxia and nutrient deprivation, enhancing metastatic spread by supporting epithelial-mesenchymal transition (EMT), tumor cell dormancy and tumor-initiating cell function, thereby stimulating angiogenesis (29). ER stress can activate the UPR, which is mediated by three ER membrane localized stress sensing proteins: Inositol-requiring enzyme 1, activating transcription factor 6 and protein kinase RNA-like ER kinase (30). Additionally, UPR activation may be tumor-supportive or suppressive depending on the intensity and duration of ER stress (31). The UPR also acts to restore ER homeostasis for cancer cell survival (32). When corrective efforts are insufficient, the cell will undergo apoptosis (33). In the current study, FNDC3B was localized to the ER. The results of the present study may therefore indicate the function of FNDC3B in ER stress and UPR.

Although FNDC3B acts as an oncogenic gene, its target genes have not been identified. However, certain studies have indicated that FNDC3B may be involved in stress granule formation-mediated ER stress (34-36). Stress granules are dense aggregations that are composed of mRNAs and proteins under conditions of stress. FNDC3B is primarily composed of fibronectin type III domains (9). FNDC3B was identified as an RNA-binding protein candidate via the interactome capture of proliferating human HeLa cells (37). When cells were challenged with ER stress, stress granules were formed (35). FNDC3B has also been identified in stress granule proteomes (34). Stress granules recruit various mRNAs and signaling proteins, including receptor for activated $\mathrm{C}$ kinase $1 /$ mitogen-activated protein kinase $14 / \mathrm{JNK}$, integrated stress response/phosphorylated-eukaryotic translation initiation factor 2A, rapamycin and Rho GTPase signaling pathways, which modulate metabolism, growth and survival (36). However, the role of FNDC3B in stress granule formation requires further elucidation.

In congruence with the current study, a previous study has indicated that FNDC3B induces and activates the PI3K/Akt signaling pathway (3). The FNDC3B co-expression genes identified in the present study were enriched in the PI3K/Akt 
A

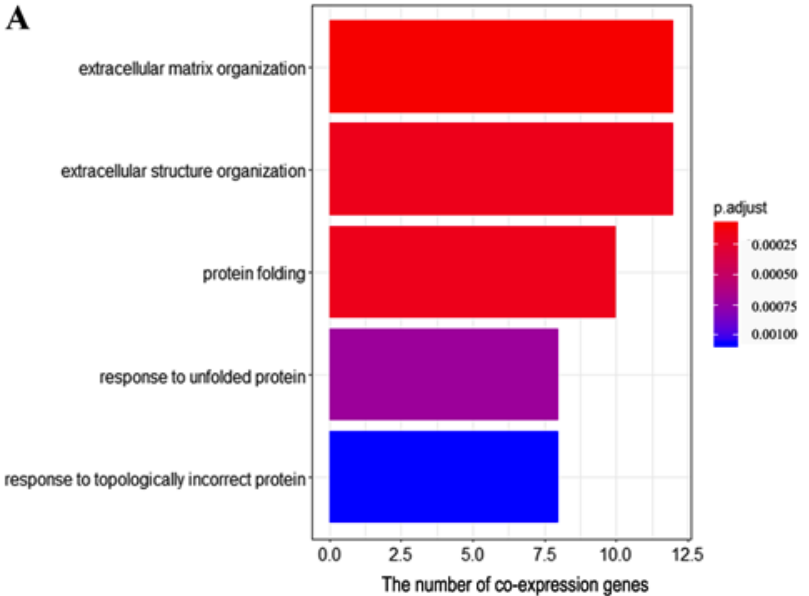

C

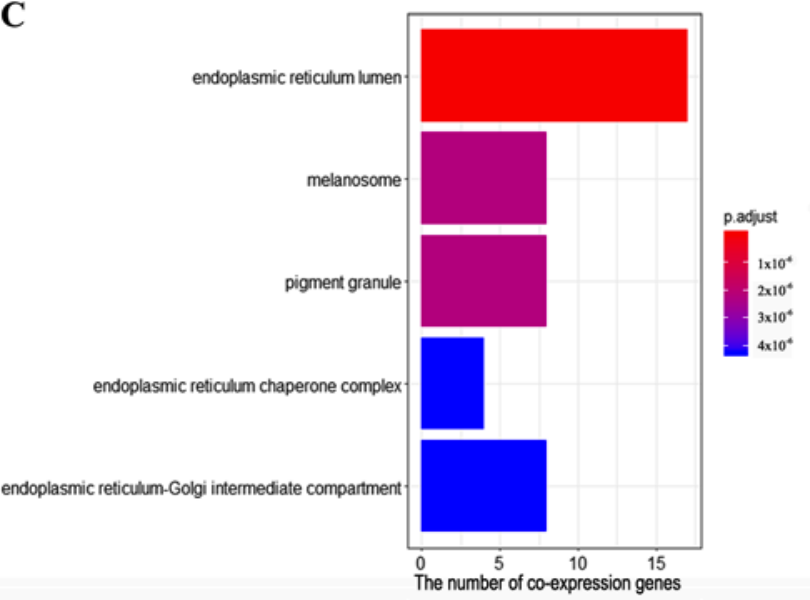

B

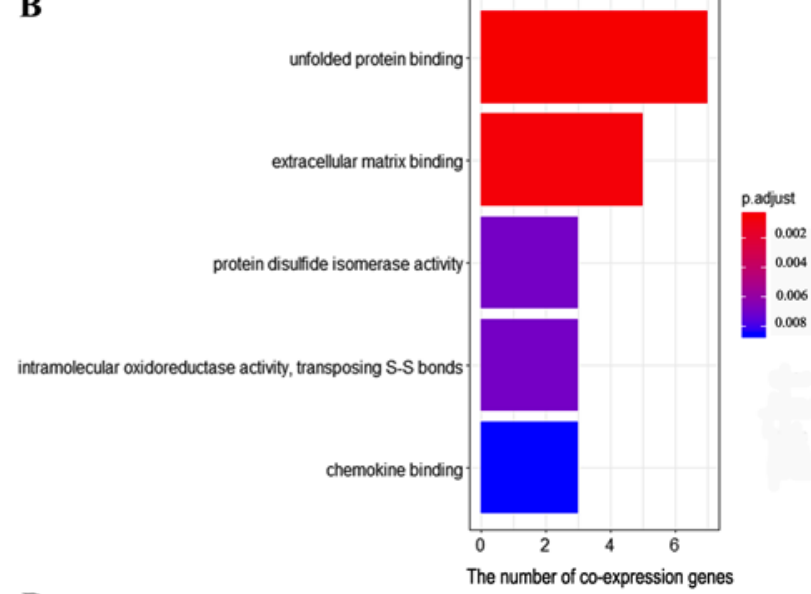

D

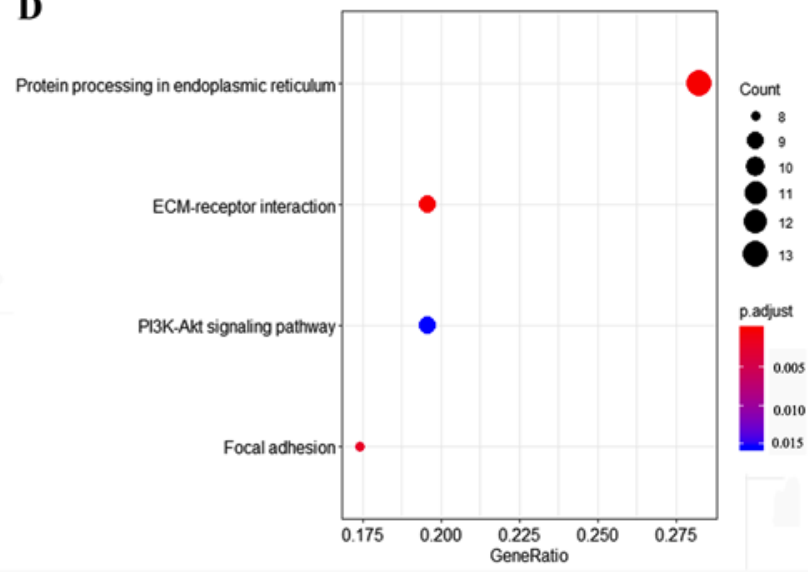

Figure 4. GO and KEGG enrichment analyses in co-expression genes associated with FNDC3B. GO terms and KEGG pathways were enriched and visualized using the clusterProfiler package in R. The P-value was adjusted using the Benjamini-Hochberg method. Enriched terms or pathways with a low or high significance are presented in blue and red, respectively. The horizontal axis in (A-C) was designed to indicate the number of co-expression genes enriched in each term, while the vertical axis indicates the enriched terms. The top five most enriched GO terms belonging to the category of (A) BP, (B) MF and (C) CC are presented. (D) Enriched KEGG pathways are presented. The label size represents the number of co-expression genes enriched in each pathway. GO, Gene Ontology; KEGG, Kyoto Encyclopedia of Genes and Genomes; FNDC3B, fibronectin type III domain containing 3B; BP, biological process; MF, molecule function; CC, cellular component.
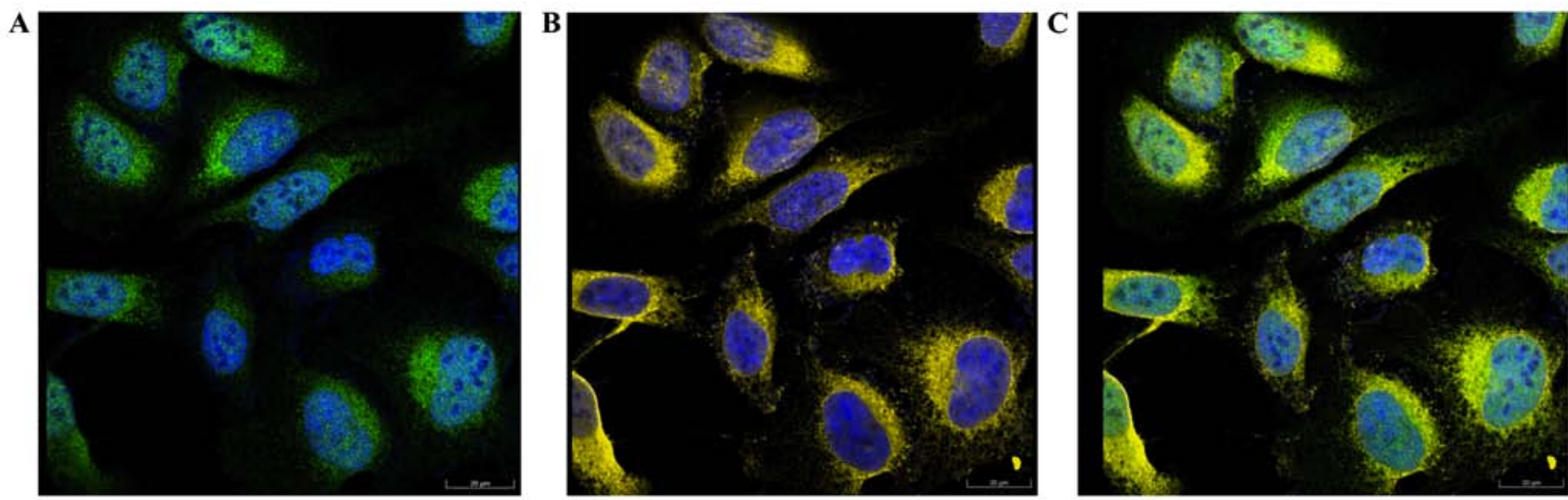

Figure 5. Cellular localization of FNDC3B determined via the Human Protein Atlas database. Immunofluorescence analysis results were obtained from the Human Protein Atlas database. FNDC3B and organelle probes are presented as different channels in the multicolor images. (A) FNDC3B was labeled with green fluorescence and nuclei were stained in blue. (B) ER was stained yellow and nuclei were stained blue. (C) Merged image of (A) and (B). FNDC3B, fibronectin type III domain containing 3B.

signaling pathway, which serves a pivotal role in tumor growth, proliferation, metabolism, motility, migration, invasion, angiogenesis, survival and autophagy (38). Considering the additional pathway enrichment of 'ECM-receptor interaction' and 'focal adhesion' determined in the current study, FNDC3B may be involved in migration and invasion. 
In conclusion, the present study revealed that FNDC3B was upregulated in cervical cancer tissue compared with normal tissue. Furthermore, elevated FNDC3B levels were associated with poor OS. Therefore, it was determined that elevated FNDC3B may be a biomarker for poor prognosis in patients with cervical cancer. Coupled with the co-expression network analysis of the current study, it was inferred that FNDC3B may serve an oncogenic role in cancer development via ER stress, UPR, cell migration and invasion. However, further studies are required to determine the exact molecular mechanism of FNDC3B in the development of cervical cancer and its potential as a novel therapeutic target.

\section{Acknowledgements}

Not applicable.

\section{Funding}

The current study was supported by the MajorNewDrugsResearch \& Development Special Project of the Ministry of Science and Technology of P.R. China (grant no. 2018ZX09303015).

\section{Availability of data and materials}

All datasets used and/or analyzed during the present study are available from the corresponding author on reasonable request.

\section{Authors' contributions}

JZ, JT and HW designed the present study. BH and JZ performed the experiments, analyzed the data and prepared the manuscript. All authors read and approved the final version of the manuscript.

\section{Ethics statement and consent to participate}

Not applicable.

\section{Patient consent for publication}

Not applicable.

\section{Competing interests}

The authors declare that they have no competing interests.

\section{References}

1. Bray F, Ferlay J, Soerjomataram I, Siegel RL, Torre LA and Jemal A: Global cancer statistics 2018: GLOBOCAN estimates of incidence and mortality worldwide for 36 cancers in 185 countries. CA Cancer J Clin 68: 394-424, 2018.

2. Cohen PA, Jhingran A, Oaknin A and Denny L: Cervical cancer. Lancet 393: 169-182, 2019.

3. Cai C, Rajaram M, Zhou X, Liu Q, Marchica J, Li J and Powers RS: Activation of multiple cancer pathways and tumor maintenance function of the $3 q$ amplified oncogene FNDC3B. Cell Cycle 11: 1773-1781, 2012.

4. Smith RA, Andrews KS, Brooks D, Fedewa SA, Manassaram-Baptiste D, Saslow D, Brawley OW and Wender RC: Cancer screening in the United States, 2018: A review of current American Cancer Society guidelines and current issues in cancer screening. CA Cancer J Clin 68: 297-316, 2018.
5. Siegel RL, Miller KD and Jemal A: Cancer statistics, 2018. CA Cancer J Clin 68: 7-30, 2018.

6. Choi YJ and Park JS: Clinical significance of human papillomavirus genotyping. J Gynecol Oncol 27: e21, 2016.

7. Tominaga K, Kondo C, Johmura Y, Nishizuka M and Imagawa M: The novel gene fad104, containing a fibronectin type III domain, has a significant role in adipogenesis. FEBS Lett 577: 49-54, 2004.

8. Nishizuka M, Kishimoto K, Kato A, Ikawa M, Okabe M, Sato R, Niida H, Nakanishi M, Osada S and Imagawa M: Disruption of the novel gene fad104 causes rapid postnatal death and attenuation of cell proliferation, adhesion, spreading and migration. Exp Cell Res 315: 809-819, 2009.

9. Lin CH, Lin YW, Chen YC, Liao CC, Jou YS, Hsu MT and Chen CF: FNDC3B promotes cell migration and tumor metastasis in hepatocellular carcinoma. Oncotarget 7: 49498-49508, 2016.

10. Noell G, Faner R and Agusti A: From systems biology to P4 medicine: Applications in respiratory medicine. Eur Respir Rev 27: pii: $170110,2018$.

11. Lin M, Ye M, Zhou J, Wang ZP and Zhu X: Recent advances on the molecular mechanism of cervical carcinogenesis based on systems biology technologies. Comput Struct Biotechnol J 17: 241-250, 2019.

12. Rhodes DR, Yu J, Shanker K, Deshpande N, Varambally R, Ghosh D, Barrette T, Pandey A and Chinnaiyan AM: ONCOMINE: A cancer microarray database and integrated data-mining platform. Neoplasia 6: 1-6, 2004.

13. Liu Y, Cui S, Li W, Zhao Y, Yan X and Xu J: PAX3 is a biomarker and prognostic factor in melanoma: Database mining. Oncol Lett 17: 4985-4993, 2019.

14. Gao J, Aksoy BA, Dogrusoz U, Dresdner G, Gross B, Sumer SO, Sun Y, Jacobsen A, Sinha R, Larsson E, et al: Integrative analysis of complex cancer genomics and clinical profiles using the cBioPortal. Sci Signal 6: pl1, 2013.

15. Cerami E, Gao J, Dogrusoz U, Gross BE, Sumer SO, Aksoy BA, Jacobsen A, Byrne CJ, Heuer ML, Larsson E, et al: The cBio cancer genomics portal: An open platform for exploring multidimensional cancer genomics data. Cancer Discov 2: 401-404, 2012.

16. Therneau T: A Package for survival analysis in S. R package version 2.38. https://CRAN.R-project.org/package=survival (2015).

17. Terry MT and Grambsch PM: Modeling survival data: Extending the cox model. Springer, New York, 2000.

18. Szklarczyk D, Gable AL, Lyon D, Junge A, Wyder S, Huerta-Cepas J, Simonovic M, Doncheva NT, Morris JH, Bork P, et al: STRING v11: Protein-protein association networks with increased coverage, supporting functional discovery in genome-wide experimental datasets. Nucleic Acids Res 47: D607-D613, 2019.

19. Shannon P, Markiel A, Ozier O, Baliga NS, Wang JT, Ramage D, Amin N, Schwikowski B and Ideker T: Cytoscape: A software environment for integrated models of biomolecular interaction networks. Genome Res 13: 2498-2504, 2003.

20. Yu G, Wang LG, Han Y and He QY: ClusterProfiler: An R package for comparing biological themes among gene clusters. OMICS 16: 284-287, 2012.

21. Uhlen M, Zhang C, Lee S, Sjöstedt E, Fagerberg L, Bidkhori G, Benfeitas R, Arif M, Liu Z, Edfors F, et al: A pathology atlas of the human cancer transcriptome. Science 357: pii: eaan2507, 2017.

22. Pyeon D, Newton MA, Lambert PF, den Boon JA, Sengupta S Marsit CJ, Woodworth CD, Connor JP,Haugen TH, Smith EM, et al: Fundamental differences in cell cycle deregulation in human papillomavirus-positive and human papillomavirus-negative head/neck and cervical cancers. Cancer Res 67: 4605-4619, 2007.

23. Scotto L, Narayan G, Nandula SV, Arias-PulidoH,Subramaniyam S, Schneider A, Kaufmann AM, Wright JD, Pothuri B, Mansukhani M and Murty VV: Identification of copy number gain and overexpressed genes on chromosome arm $20 \mathrm{q}$ by an integrative genomic approach in cervical cancer: Potential role in progression. Genes Chromosomes Cancer 47: 755-765, 2008.

24. Vitale A and Denecke J: The endoplasmic reticulum-gateway of the secretory pathway. Plant Cell 11: 615-628, 1999.

25. Jain BP: An overview of unfolded protein response signaling and its role in cancer. Cancer Biother Radiopharm 32: 275-281, 2017.

26. Wang $M$ and Kaufman RJ: The impact of the endoplasmic reticulum protein-folding environment on cancer development. Nat Rev Cancer 14: 581-597, 2014.

27. Wang M, Law ME, Castellano RK and Law BK: The unfolded protein response as a target for anticancer therapeutics. Crit Rev Oncol Hematol 127: 66-79, 2018. 
28. Taguchi Y, Horiuchi Y, Kano F and Murata M: Novel prosurvival function of Yip1A in human cervical cancer cells: Constitutive activation of the IRE1 and PERK pathways of the unfolded protein response. Cell Death Dis 8: e2718, 2017.

29. Cubillos-Ruiz JR, Bettigole SE and Glimcher LH: Tumorigenic and immunosuppressive effects of endoplasmic reticulum stress in cancer. Cell 168: 692-706, 2017.

30. Walter P and Ron D: The unfolded protein response: From stress pathway to homeostatic regulation. Science 334: 1081-1086, 2011.

31. Wang M and Kaufman RJ: Protein misfolding in the endoplasmic reticulum as a conduit to human disease. Nature 529: 326-335, 2016.

32. Tashiro E: Screening and identification of inhibitors of endoplasmic reticulum stress-induced activation of the IRE1a-XBP1 branch. J Antibiot (Tokyo): Aug 9, 2019 (Epub ahead of print).

33. Maurel M, McGrath EP, Mnich K, Healy S, Chevet E and Samali A: Controlling the unfolded protein response-mediated life and death decisions in cancer. Semin Cancer Biol 33: 57-66, 2015.

34. Jain S, Wheeler JR, Walters RW, Agrawal A, Barsic A and Parker R: ATPase-modulated stress granules contain a diverse proteome and substructure. Cell 164: 487-498, 2016.
35. Namkoong S, Ho A, Woo YM, Kwak H and Lee JH: Systematic characterization of stress-induced RNA granulation. Mol Cell 70: 175-187 e8, 2018

36. Kedersha N, Ivanov P and Anderson P: Stress granules and cell signaling: More than just a passing phase? Trends Biochem Sci 38: 494-506, 2013.

37. Castello A, Fischer B, Eichelbaum K, Horos R, Beckmann BM, Strein C, Davey NE, Humphreys DT, Preiss T, Steinmetz LM, et al: Insights into RNA biology from an atlas of mammalian mRNA-binding proteins. Cell 149: 1393-1406, 2012.

38. McAuliffe PF, Meric-Bernstam F, Mills GB and Gonzalez-Angulo AM: Deciphering the role of PI3K/Akt/mTOR pathway in breast cancer biology and pathogenesis. Clin Breast Cancer 10 (Suppl 3): S59-S65, 2010.

This work is licensed under a Creative Commons Attribution-NonCommercial-NoDerivatives 4.0 International (CC BY-NC-ND 4.0) License. 